\title{
Risk stratification by pre-operative cardiopulmonary exercise testing improves outcomes following elective abdominal aortic aneurysm surgery: a cohort study
}

Stephen J Goodyear ${ }^{1 *}$, Heng Yow ${ }^{1}$, Mahmud Saedon ${ }^{1,2}$, Joanna Shakespeare ${ }^{1}$, Christopher E Hill', Duncan Watson ${ }^{1}$, Colette Marshall', Asif Mahmood ${ }^{1}$, Daniel Higman ${ }^{1}$ and Christopher HE Imray ${ }^{1,2}$

\begin{abstract}
Background: In 2009, the NHS evidence adoption center and National Institute for Health and Care Excellence (NICE) published a review of the use of endovascular aneurysm repair (EVAR) of abdominal aortic aneurysms (AAAs). They recommended the development of a risk-assessment tool to help identify AAA patients with greater or lesser risk of operative mortality and to contribute to mortality prediction.

A low anaerobic threshold (AT), which is a reliable, objective measure of pre-operative cardiorespiratory fitness, as determined by pre-operative cardiopulmonary exercise testing (CPET) is associated with poor surgical outcomes for major abdominal surgery. We aimed to assess the impact of a CPET-based risk-stratification strategy upon perioperative mortality, length of stay and non-operative costs for elective (open and endovascular) infra-renal AAA patients.

Methods: A retrospective cohort study was undertaken. Pre-operative CPET-based selection for elective surgical intervention was introduced in 2007. An anonymized cohort of 230 consecutive infra-renal AAA patients (2007 to 2011) was studied. A historical control group of 128 consecutive infra-renal AAA patients (2003 to 2007) was identified for comparison.

Comparative analysis of demographic and outcome data for CPET-pass (AT $\geq 11 \mathrm{ml} / \mathrm{kg} / \mathrm{min}$ ), CPET-fail (AT < $11 \mathrm{ml} /$ $\mathrm{kg} / \mathrm{min}$ ) and CPET-submaximal (no AT generated) subgroups with control subjects was performed. Primary outcomes included 30-day mortality, survival and length of stay (LOS); secondary outcomes were non-operative inpatient costs.
\end{abstract}

Results: Of 230 subjects, 188 underwent CPET: CPET-pass $n=131$, CPET-fail $n=35$ and CPET-submaximal $n=22$. When compared to the controls, CPET-pass patients exhibited reduced median total LOS (10 vs 13 days for open surgery, $n=74, P<0.01$ and 4 vs 6 days for EVAR, $n=29, P<0.05$ ), intensive therapy unit requirement ( 3 vs 4 days for open repair only, $P<0.001)$, non-operative costs ( $£ 5,387$ vs $£ 9,634$ for open repair, $P<0.001)$ and perioperative mortality (2.7\% vs $12.6 \%$ (odds ratio: 0.19$)$ for open repair only, $P<0.05$ ). CPET-stratified (open/endovascular) patients exhibited a mid-term survival benefit $(P<0.05)$.

Conclusion: In this retrospective cohort study, a pre-operative AT $>11 \mathrm{ml} / \mathrm{kg} / \mathrm{min}$ was associated with reduced perioperative mortality (open cases only), LOS, survival and inpatient costs (open and endovascular repair) for elective infra-renal AAA surgery.

Keywords: AAA, Abdominal aortic aneurysm, CPET, Cardiopulmonary exercise test, Clinical outcomes

\footnotetext{
*Correspondence: drgoodyear@hotmail.com

1 University Hospitals Coventry and Warwickshire NHS Trust, Clifford Bridge Road, Coventry CV2 2DX, UK

Full list of author information is available at the end of the article
} 


\section{Background}

It is more important to know what sort of person has a disease than to know what sort of disease a person has (Hippocrates, 460 to $370 \mathrm{BC}$ ).

Open abdominal aortic aneurysm (AAA) surgery places substantial metabolic demands upon patients during the perioperative period. These result from increased energy requirements necessary for wound healing [1], hemostasis, ventilation, significant intra-operative hemodynamic [2,3] and acid/base fluctuations in addition to the catecholamine stress response to surgery [4-6]. Failure of the cardiorespiratory system to meet these increased metabolic requirements of patients undergoing major abdominal surgery may lead to avoidable cardiorespiratory morbidity and mortality [7-10]. Aortic surgery is associated with a high $(\geq 5 \%)$ combined incidence of cardiac death and non-fatal myocardial infarction [11]. An individual's functional status has been shown to be reliably predictive of perioperative and long-term cardiac events following non-cardiac surgery [11], which can be derived from an assessment of their ability to perform activities of daily living $[12,13]$. Functional capacity (a numeric measure of functional status) can be expressed in metabolic equivalent (MET) levels; the oxygen consumption $\left(\mathrm{VO}_{2}\right)$ of a $70-\mathrm{kg}$, 40-year-old man in a resting state is $3.5 \mathrm{ml} / \mathrm{kg} / \mathrm{min}$ or $1 \mathrm{MET}$ [11]. The American College of Cardiology and American Heart Association (ACC/AHA) guidelines for perioperative assessment states that patients able to demonstrate a functional capacity of 4 METS may safely proceed to surgery without further cardiac assessment [14]. This equates to the ability to climb a flight of stairs or run a short distance. However, subjective assessment of functional status by clinicians for patients undergoing AAA repair, can be easily confounded and lacks prognostic accuracy [15-17], identifying a potential role for objective testing.

Static pre-operative tests of cardiac function, such as resting left ventricular ejection fraction, correlate poorly with cardiorespiratory (physical) fitness [18,19], whilst dynamic tests such as dobutamine stress echocardiography and stress electrocardiogram (ECG) testing do not measure respiratory function and global oxygen delivery simultaneously. Cardiopulmonary exercise testing (CPET) allows the objective quantification of the level at which end-organ oxygen demand exceeds delivery [20] (the functional reserve) and may be safely performed in high-risk populations $[7,15,21]$. The transition point at which the production of $\mathrm{CO}_{2}$ exceeds $\mathrm{VO}_{2}$ is known as the anaerobic threshold (AT) and can be determined by gaseous exchange measurement during CPET [20]. More simply, the AT is the work rate at which an individual's cardiorespiratory system fails to deliver sufficient oxygen to maintain aerobic respiration, mandating usage of an anaerobic substrate. AT is recognized as a reliable measure of preoperative fitness in AAA patient populations [22].

Older et al. demonstrated that a critical AT $\geq$ $11 \mathrm{ml} / \mathrm{kg} / \mathrm{min}$ for elderly subjects was associated with $0.8 \%$ perioperative mortality rate in major abdominal surgery, compared to $18 \%$ in individuals below this level [23]. In a further study, the same center identified a less intensive perioperative care requirement and reduced cardiovascular and all-cause mortality for elderly (major surgical) patients with $\mathrm{AT} \geq 11$, when compared to individuals below this threshold [24]. Additional work has shown anaerobic threshold $>11$ to be associated with fewer short-term complications and hence a shorter length of inpatient stay (LOS) following major abdominal surgery [25]. More contemporary evidence highlights other variables obtained during CPET to be at least as valuable (as AT) in predicting short- and mid-term outcomes in elective AAA surgery $[7,26]$. This is supported by a recent finding that AT $<10.2 \mathrm{ml} / \mathrm{kg} / \mathrm{min}$ and peak oxygen consumption $\left(\mathrm{VO}_{2}\right.$ peak $)<15 \mathrm{ml} / \mathrm{kg} / \mathrm{min}$ were associated with increased 30- and 90-day mortality following AAA surgery [27].

The aim of elective surgery for AAA is to prolong the survival of patients. However, there is an increasing awareness that many of these individuals have significant and potentially life-threatening cardiorespiratory comorbidities [26]. Proactive screening for AAA reduces the prevalence of aneurysm-related mortality [28] and surgical intervention when the AAA $\geq 5.5 \mathrm{~cm}$ anteriorposterior (AP) diameter is appropriate [29] in units that have low perioperative complication rates. Large multicenter trials such as EVAR-1 have published 30-day mortality rates of $5.3 \%$ for open AAA repair [30]. However, the Vascular Society of Great Britain and Ireland's (VSGBI) quality improvement framework (QIF) target of $3.5 \%$ by 2013 suggests that this could be improved further [31].

Pre-operative CPET was introduced for elective aneurysm surgery at University Hospitals Coventry and Warwickshire (UHCW) NHS Trust in 2007 in response to a 30 -day mortality rate of $12.6 \%$ for open elective surgery, determined by internal audit and following an invited review by the VSGBI. These data reflected the hospitals' all-comers policy to elective aneurysm surgery, offering operative repair to many individuals of equivocal cardiovascular health, who may have been declined intervention in vascular units with more stringent perioperative selection. The observed mortality rate for unselected patients prior to 2007 is comparable with the findings of Carlisle and Swart (30-day mortality rate: 9\%) [26], who studied infra-renal AAA outcomes during a similar era. Whilst 12.6\% 30-day mortality for unstratified (pre-CPET era) open AAA surgery appears unacceptably high at first glance, there is a significant moral dilemma to be ad- 
dressed when consideration is given to the overall mortality rate for ruptured AAA, generally accepted as 90\% [32]. Nevertheless, these mortality data fell beyond the established range quoted in the literature [33] and a trustwide guideline of pre-operative CPET-stratification-based selection (for all elective AAA patients) was introduced, to facilitate ongoing AAA intervention at the established diameter of $5.5 \mathrm{~cm}$, on a risk-benefit basis. Conservative management was offered to individuals considered to be at high risk of perioperative mortality following stratification, based upon an extrapolation of the 2007 Carlisle and Swart data [26].

This study aimed to assess the outcomes of pre-operative CPET-stratification on the duration of postoperative inpatient stay, intensive therapy unit (ITU) usage, end-organ support, 30-day mortality rates and longer-term survival following elective open and endovascular infra-renal AAA repair.

\section{Methods}

This study is a retrospective, anonymized, single-center, cohort study performed at UHCW NHS Trust, a centralized vascular unit serving a population of 950,000. A review of the study proposal was undertaken by the trust's Research and Development department; ethical approval was deemed unnecessary, based upon National Research Ethics Service guidance [34].

From November 2007, all patients considered for elective AAA repair $(\mathrm{AAA} \geq 5.5 \mathrm{~cm})$ surgery were recommended pre-operative CPET. An evidence-based minimum anaerobic threshold of $11.0 \mathrm{ml} / \mathrm{kg} / \mathrm{min}$ was selected to identify individuals with adequate cardiopulmonary reserves who would be able to tolerate general anesthesia and open abdominal surgery, with acceptable perioperative mortality rates (the CPET-pass subgroup). These individuals were offered the option of endovascular aneurysm repair (EVAR), if anatomically applicable, or open AAA repair. Individuals attaining an $\mathrm{AT}<11 \mathrm{ml} / \mathrm{kg} / \mathrm{min}$ (the CPET-fail subgroup) were counseled regarding conservative management if EVAR (a less cardiovascularly challenging procedure) was not anatomically feasible. CPET-fail patients with unfavorable anatomy for infra-renal EVAR who requested open repair (rather than expectant management), were permitted to proceed following careful discussion and documentation of the perceived increased mortality risk. Individuals who were unable to demonstrate AT during CPET due to mechanical co-morbidities, suboptimal effort, suboptimal compliance with the investigation or ECG changes at minimal exertion (the CPET-submaximal subgroup) were managed as per the CPET-fail subgroup.

\section{Data collection and analysis}

The data for 230 consecutive infra-renal AAA $(\geq 5.5 \mathrm{~cm}$ AP diameter) patients considered for elective surgery between November 2007 and July 2011 (the CPET era) were studied. A control group of 128 consecutive individuals who underwent open or endovascular (infrarenal) AAA repair between January 2003 and October 2007 (the pre-CPET era) were identified for comparison. Patients diagnosed with thoracoabdominal or suprarenal aneurysms were excluded from the data collection, in addition to individuals who had undergone repairs of ruptured or urgent (symptomatic, non-ruptured) AAA.

Individuals were identified (with permission) by the Department of Clinical Coding using KMR1 diagnoses and procedures. To ensure completeness of data, results were cross-referenced with (computerized and written) operating theatre registries, the Dr Foster national outcomes database and the results of an internal audit of mortality/ morbidity for elective AAA patients. Cardiopulmonary exercise testing data for CPET-era patients were collected (with full permission of the Department of Respiratory Physiology) from the UHCW CPET database.

Demographic and outcome data were identified by a systematic review of the hospitals' Clinical Results Reporting System (CRRS) and the patient case notes. Data loss was minimized by cross-referencing, using anonymized patient identification (PID) numbers, with the ITU patient digital registry and the CPET database. Information relating to length of ITU stay and the number of end organs supported was obtained from the ITU patient digital registry. Mortality data were sourced (with permission) from CRRS, the hospitals' Bereavement Services Department and by liaison with primary care providers. Survival was calculated from the date of intervention until death or censorship, in days. For individuals managed conservatively (CPET era), survival was calculated from the date of CPET until death or censorship. Survival could be observed for up to eight years in the control cohort and a maximum of four years for the CPET-era cohort, within the constraints of this study and this is regarded as mid-term survival. Tariffs for ITU and ward stays were obtained from the Department of Health Report 2010/2011 for UHCW via the Finance Section of the Information Services department. ITU cost data were calculated on an individual basis. A variable tariff applied, based upon the total number of end organs postoperatively supported (Table 1) multiplied by the duration of ITU stay. Ward costs were calculated by multiplication of a standard tariff (Table 1) by duration of ward stay. Financial analysis did not consider the costs of staff, equipment and consumables, which are essentially constant throughout NHS organizations offering similar interventions.

\section{Surgical technique}

All elective infra-renal AAA repairs were discussed and planned within a multidisciplinary team. Open aneurysm 


\begin{tabular}{|c|c|c|}
\hline Location & Discriminator & Cost per day $(£)$ \\
\hline CPET & One-off tariff & 150 \\
\hline \multirow[t]{2}{*}{ Surgical ward } & Influenceable costs & 110 \\
\hline & Fully absorbed costs & 250 \\
\hline \multirow[t]{7}{*}{ ITU } & 0 organs supported & 260 \\
\hline & 1 organ supported & 769 \\
\hline & 2 organs supported & 1106 \\
\hline & 3 organs supported & 1386 \\
\hline & 4 organs supported & 1511 \\
\hline & 5 organs supported & 1568 \\
\hline & 6 organs supported & 1638 \\
\hline
\end{tabular}

repairs were performed by a consultant vascular surgeon, or a consultant-supervised higher surgical trainee, using a transperitoneal inlay repair with knitted Dacron graft prostheses. Endovascular aneurysm repairs were planned and performed by a consultant vascular surgeon and consultant interventional radiologist. The EVAR devices used were the Cook Zenith (Cook, Brisbane, Australia) endovascular system, Medtronic Endurant ${ }^{\circ}$ (Medtronic, Minneapolis, MN, USA) and Lombard Aorfix $^{\mathrm{mi}}$ (Lombard Medical, Oxfordshire, UK).

\section{Cardiopulmonary exercise testing}

Prior to testing, the patient's body mass index (BMI) was determined by measurement of height and weight. Resting spirometry was performed to measure forced expiratory volume per second $\left(\mathrm{FEV}_{1}\right)$ and forced vital capacity (FVC), from which $\mathrm{FEV}_{1} / \mathrm{FVC}$ ratios were calculated. Predicted $\mathrm{FEV}_{1}$ and FVC values were derived as a function of age, height, ethnicity and gender (calculated by the CPET software - see below). $\mathrm{FEV}_{1}$ data were used to assess an individual's maximum predicted ventilation. The patient's weight and predicted maximum oxygen uptake $\left(\mathrm{VO}_{2} \max \right)$ were used to calculate the individually required work rate on the cycle ergometer. Patients were subsequently attached to a 12-lead ECG and a form-fitting face-mask connected to a metabolic cart with protocol specific software (Viasprint Ergometer and MasterScreen CPX software v5.21.0.60, CareFusion Corporation, CA, USA). Patients were initially made to pedal for an unloaded phase (work rate $0 \mathrm{~W}$ at $50 \mathrm{rpm}$ for $3 \mathrm{~min}$ ) followed by a ramped phase requiring a constant $70 \mathrm{rpm}$ against increasing resistance until they reached their peak oxygen uptake $\left(\mathrm{VO}_{2}\right.$ peak). The test could be stopped at any point during the test protocol due to patient fatigue, presence of ischemic ECG changes, chest pain or if the maximum heart rate was achieved. The AT was derived using the V-slope method as described by Beaver et al. [35].

\section{Statistical analysis}

All data were tabulated using a Microsoft Excel ${ }^{\circ}$ spreadsheet (Microsoft, CA, USA) and statistical analyses performed using Graphpad Prism4 $4^{\circ}$ (Graphpad, La Jolla, CA, USA). The normality of data was assessed with the D'Agostino and Pearson omnibus normality test. Nonparametric unpaired data were analyzed using the Mann-Whitney $U$ test or Kruskal-Wallace analysis of variance (ANOVA), whilst categorical variables were analyzed using the chi-squared test or Fisher's exact test. Parametric data were assessed with a Student's t-test or one-way ANOVA. Survival data were evaluated by Kaplan-Meier curves. A $P$ value of less than 0.05 was considered significant.

\section{Results}

Of 128 control subjects in the pre-CPET era, there were $103(80.5 \%)$ open AAA repairs and 25 (19.5\%) EVARs. Following introduction of CPET, 230 consecutive subjects with elective infra-renal AAA were studied. Operated cases included open repair in 100 (59.2\%) patients and EVAR in 69 (40.8\%), representing a significant increase in the proportion of endovascular cases $(P<0.001)$. A further 61 individuals did not have intervention.

Composition of the CPET-era open surgery, EVAR and no intervention groups, with respect to CPET outcomes, are demonstrated in Figure 1.

\section{CPET subgroups}

Of the 230 subjects identified in the CPET era, 188 underwent CPET. Tested individuals were stratified by their anaerobic threshold into three cohorts: CPET-pass $(n=131, \mathrm{AT} \geq 11.0 \mathrm{ml} / \mathrm{kg} / \mathrm{min})$, CPET-fail $(n=35$, AT $<11.0)$ and CPET-submaximal $(n=22$, unable to generate an AT). Of the patients, 42 were not referred for CPET (Figure 1).

Table 2 shows demographic data for pre-CPET era controls and CPET-era subgroups. Control subjects were of comparable age (median age: 74.0 years, $95 \%$ CI: 71.9 to 74.4) and equivalent aneurysm size (median aneurysm diameter: $6.3 \mathrm{~cm}, 95 \% \mathrm{CI}: 6.5$ to 6.9$)$ to those in the CPET-stratified subgroups. BMI was infrequently recorded during the pre-CPET era rendering these data unsuitable for comparison. However, the median age was significantly higher in the CPET-submaximal group compared to CPET-pass patients $(P<0.01)$, the untested cohort $(P<0.01)$ and the pre-CPET cohort $(P<0.01)$. In addition, BMI was significantly lower in the CPET-pass cohort than the CPET-fail group $(P<0.05)$.

\section{Length of inpatient stay}

The median length of inpatient stay was significantly longer for open AAA surgery than EVAR in both the 


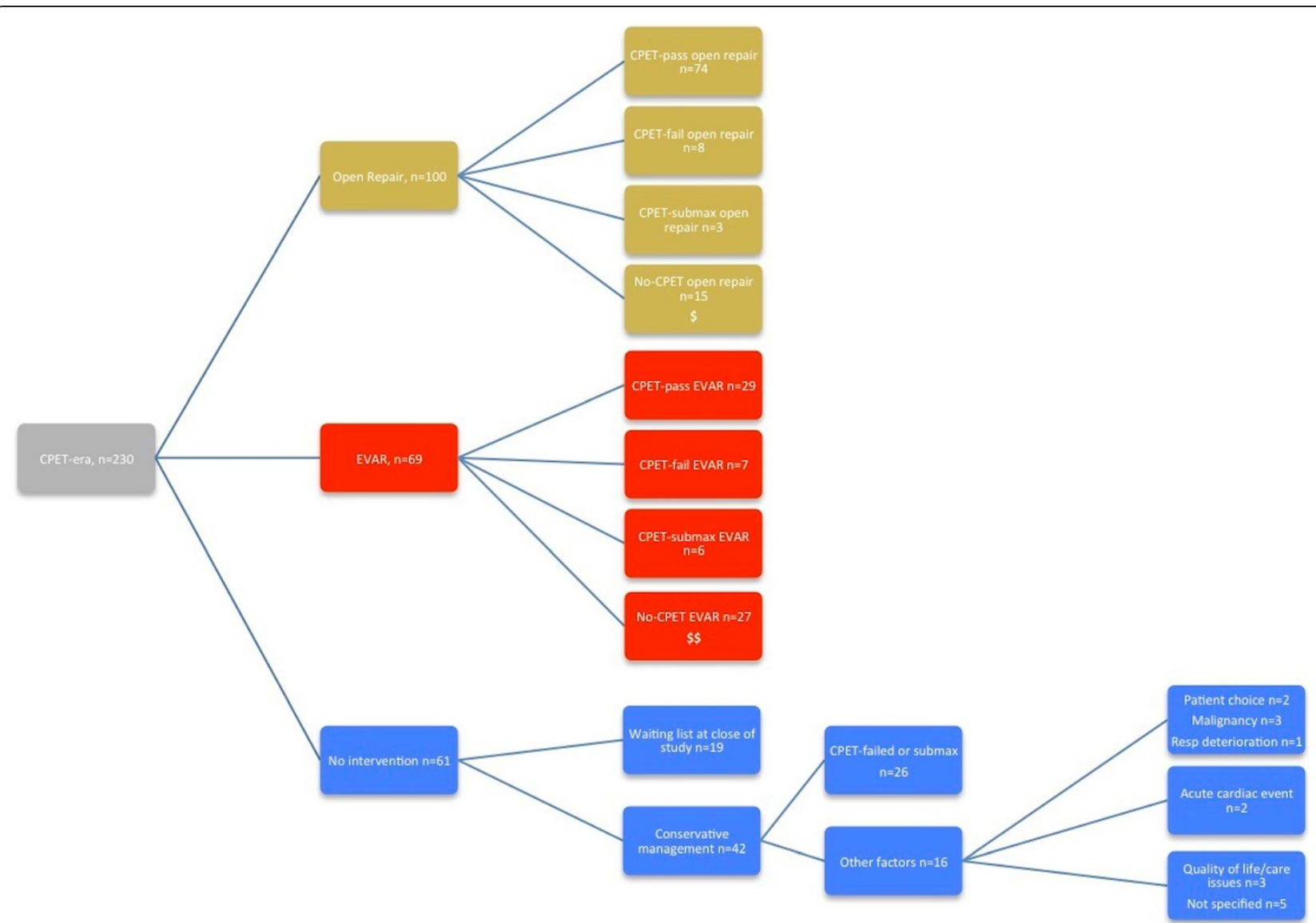

Figure $1 \mathrm{~A}$ consort-type diagram representing the composition of the CPET-era cohort by management type and their subgroups by CPET stratification. \$ and \$ indicate the 42 individuals who were not referred for CPET within this cohort.

pre-CPET (open surgery: 13 days, $95 \%$ CI: 13.9 to 19.0 ; EVAR: 6 days, $95 \%$ CI: 4.3 to 8.3 days; $P<0.001)$ and post-CPET (open surgery: 10 days, $95 \%$ CI: 10.3 to 13.5 ; EVAR: 4 days, 95\% CI: 4.6 to 6.7 days; $P<0.001)$ cohorts.

\section{Open surgery}

The length of inpatient stay following open AAA surgery in the CPET era (median: 10 days, 95\% CI: 10.3 to 13.5 ) was shorter than that in the pre-CPET era (median: 13 days, $95 \%$ CI: 13.9 to $19.0 ; P<0.001)$ principally due to the reduced duration of stay seen in the CPET-pass subgroup (Table 3, Figure 2a).

\section{EVAR}

The length of inpatient stay following EVAR in the CPET era (median: 4.0 days, 95\% CI: 4.6 to 6.7) was shorter than that in the pre-CPET era (median: 6.0 days, 95\% CI: 5.3 to $8.6 ; P<0.05$ ), due to the reduced duration of stay seen in the CPET-pass subgroup (Table 3, Figure $2 b)$.

\section{Duration of ITU stay \\ Open surgery}

The length of ITU stay was reduced in the CPET era compared to pre-CPET controls (CPET era: 3 days, 95\% CI: 3.2 to 4.4 , pre-CPET: 4 days, $95 \%$ CI: 5.5 to

Table 2 Demographic data for CPET-era elective infra-renal AAA patients compared to pre-CPET era controls

\begin{tabular}{|c|c|c|c|c|c|c|c|c|c|c|}
\hline & \multirow{2}{*}{\multicolumn{2}{|c|}{$\begin{array}{l}\text { Pre-CPET era } \\
(n=128)\end{array}$}} & \multicolumn{6}{|c|}{ Elective AAA patients - CPET performed $(n=188)$} & \multirow{2}{*}{\multicolumn{2}{|c|}{ No CPET $(n=42)$}} \\
\hline & & & \multicolumn{2}{|c|}{ CPET-pass $(n=131)$} & \multicolumn{2}{|c|}{ CPET-fail $(n=35)$} & \multicolumn{2}{|c|}{ CPET-submaximal $(n=22)$} & & \\
\hline & Median & $95 \% \mathrm{Cl}$ & Median & $95 \% \mathrm{Cl}$ & Median & $95 \% \mathrm{Cl}$ & Median & $95 \% \mathrm{Cl}$ & Median & $95 \% \mathrm{Cl}$ \\
\hline Age (years) & $74.0\left(^{* *}\right)$ & 71.9 to 74.4 & $74.0\left({ }^{* *}\right)$ & 72.1 to 74.7 & 75 & 73.1 to 78.3 & 80.5 & 76.7 to 81.4 & $72.5(* *)$ & 70.1 to 74.8 \\
\hline BMI $\left(\mathrm{kg} / \mathrm{m}^{2}\right)$ & N/A & N/A & $27.3\left({ }^{*}\right)$ & 26.8 to 28.2 & 30.0 & 27.6 to 31.4 & 27.6 & 25.7 to 31.3 & N/A & N/A \\
\hline Aneurysm size $(\mathrm{cm})$ & 6.3 & 6.5 to 6.9 & 6.1 & 6.2 to 6.6 & 6.1 & 6.0 to 6.7 & 6.3 & 6.0 to 6.9 & 5.9 & 5.9 to 6.5 \\
\hline
\end{tabular}

${ }^{*} P<0.05,{ }^{* *} P<0.01$; significance when compared to the figure highlighted in bold for the given row. 
Table 3 Mann-Whitney $U$ comparison of length of stay for open and endovascular AAA repairs

\begin{tabular}{|c|c|c|c|c|c|}
\hline Cohort & & $\begin{array}{l}\text { Median total length of } \\
\text { stay (days) }(95 \% \mathrm{Cl})\end{array}$ & $P$ value & $\begin{array}{l}\text { Median length of ITU } \\
\text { stay (days) }(95 \% \mathrm{Cl})\end{array}$ & $P$ value \\
\hline \multicolumn{6}{|l|}{ Open surgery } \\
\hline Pre-CPET $(n=103)$ & & 13 (13.9 to 19.0$)$ & & $4(5.5$ to 11.2$)$ & \\
\hline \multirow[t]{5}{*}{ CPET era $(n=100)$} & CPET-era open $(100 / 100)$ & $10(10.3$ to 13.5$)$ & $P<0.001$ & 3 (3.2 to 4.4$)$ & $P<0.01$ \\
\hline & CPET-pass (74/100) & $10(10.6$ to 14.9$)$ & $P<0.01$ & $3(2.9$ to 4.3$)$ & $P<0.001$ \\
\hline & CPET-fail (8/100) & 11.5 (8.6 to 3.9$)$ & $P=0.25$ & 4.95 (2.1 to 8.4$)$ & $P=0.88$ \\
\hline & CPET-submaximal (3/100) & $11(-5.4$ to 22.0$)$ & $P=0.18$ & $11(-5.3$ to 22.0$)$ & $P=0.59$ \\
\hline & No-CPET $(15 / 100)$ & $8(6.6$ to 11.1$)$ & $P<0.001$ & 5 (3.1 to 6.1$)$ & $P=0.82$ \\
\hline \multicolumn{6}{|l|}{$E V A R^{a}$} \\
\hline Pre-CPET $(n=25)$ & & $6(5.3$ to 8.6$)$ & & N/A & N/A \\
\hline \multirow[t]{5}{*}{ CPET era $(n=69)$} & CPET-era EVAR (69/69) & 4 (4.6 to 6.7$)$ & $P<0.05$ & $\mathrm{~N} / \mathrm{A}$ & $\mathrm{N} / \mathrm{A}$ \\
\hline & CPET-pass (29/69) & $4(3.6$ to 5.7$)$ & $P<0.05$ & N/A & N/A \\
\hline & CPET-fail (7/69) & $4(2.5$ to 8.1$)$ & $P=0.23$ & $\mathrm{~N} / \mathrm{A}$ & N/A \\
\hline & CPET-submaximal (6/69) & $4(0$ to 14.3$)$ & $P=0.56$ & N/A & N/A \\
\hline & No-CPET (27/69) & $4(4.4$ to 8.8$)$ & $P=0.14$ & N/A & N/A \\
\hline
\end{tabular}

${ }^{\mathrm{a}}$ Insufficient EVAR patients required ITU management for reasonable comparison.

11.2; $P<0.01)$, reflected only by the CPET-pass subgroup (Table 3 ).

\section{EVAR}

Too few EVAR patients required ITU care in the preCPET or CPET-era groups for meaningful statistical comparison.

\section{Total non-operative inpatient costs}

\section{Open surgery}

Total non-operative (fully absorbed) costs of inpatient stay was significantly lower for the CPET-era cohort (mean: $£ 5,229,95 \% \mathrm{CI}: 4,452$ to 6,$006 ; P<0.001$ ) compared with pre-CPET controls (mean: $£ 9,637,95 \% \mathrm{CI}$ : $7,768$ to 11,510$)$. This trend is reflected only by the CPET-pass subgroup (mean: $£ 5,387,95 \% \mathrm{CI}: 4,382$ to 6,392; $P<0.001$ ) (Figure 3 ). This is principally due to the reduced duration of ITU requirement (Table 3) and median number of end organs requiring support (preCPET: 2 organs, 95\% CI 1.9 to 2.4; CPET-pass: 1 organ 95\% CI 1.0 to $1.5 ; P<0.001)$ in the CPET-pass subgroup.

The cost benefit is maintained when calculations are repeated using influenceable ward costs (pre-CPET: $£ 8,203$ c.f. CPET-era: $£ 4,071 ; P<0.001$, CPET-pass subgroup: $£ 4,068 ; P<0.001)$.

\section{EVAR}

Non-operative costs are considered a function of total length of stay due to the minimal requirement for ITU in both the pre-CPET and CPET-era cohorts (Table 3).

\section{Total 30-day mortality} Open surgery

Total 30-day mortality for elective open surgery in the preCPET era was significantly higher than following the introduction of CPET stratification (pre-CPET 30-day mortality: $12.6 \%$, post-CPET 30 -day mortality: $4.0 \% ; P<0.05$ ). These findings are reflected only by those in the CPET-pass subgroup (Table 4).

\section{EVAR}

No significant difference was demonstrated in 30-day mortality following endovascular repair between the pre-CPET and CPET-era cohorts (pre-CPET 30-day mortality: 0\%, CPET-era 30-day mortality: $1.4 \% ; P=1.00)$.

\section{Survival}

Kaplan-Meier survival analysis demonstrated reduced mid-term survival (from surgery, or from CPET for conservatively managed patients, to censorship at closure of the study) for pre-CPET EVAR and open AAA repair patients (logrank test: $P<0.05$; Figure $4 \mathrm{a}$ ) compared to the respective operated cohorts following CPET stratification. Median survival for pre-CPET era open AAA repairs was 2,640 days (7.23 years); however, median survival of the other subgroups could not be calculated for the period studied. The mortality-rate trends were confirmed by linear regression and remained significant $(P<0.001)$.

Survival analysis comparison between pre-CPET EVAR patients and CPET-stratified conservatively managed individuals showed no significant difference (Figure 4b) at 45 months (logrank test: $P=0.96$ ). 

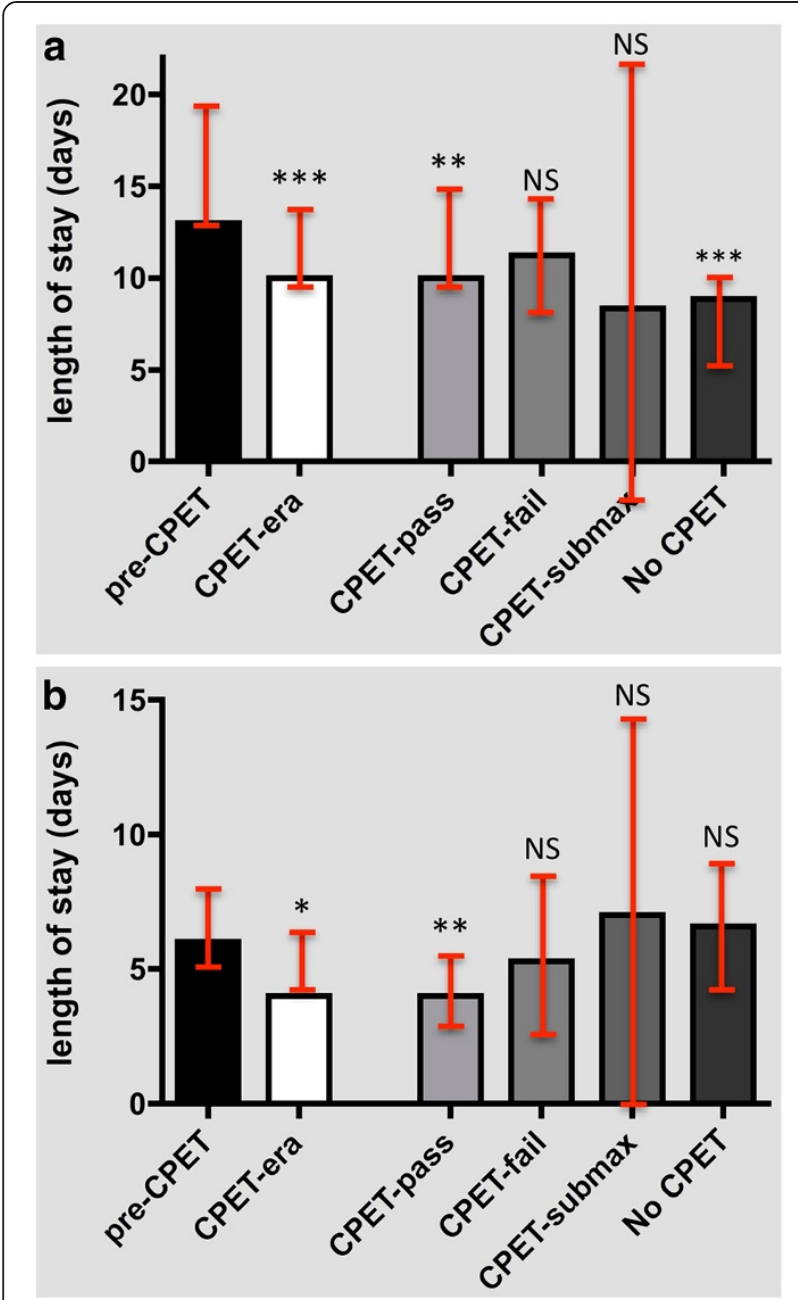

Figure 2 Length of inpatient stay. (a) Mann-Whitney $U$ analysis of total (median) length of inpatient stay for open AAA patients in the pre- and post-CPET eras. The four bars on the right represent CPET-stratification outcomes. ${ }^{* *} P<0.01$; ${ }^{* *} P<0.001$. (b) Mann-Whitney $U$ analysis of total (median) length of inpatient stay for EVAR patients in the pre- and post-CPET eras. The four bars on the right represent CPET-stratification outcomes. ${ }^{*} P<0.05$; ${ }^{*} P<0.01$. CPET: cardiopulmonary exercise testing; EVAR: endovascular aneurysm repair; NS: not significant.

Similarly, survival analysis comparison between preCPET open AAA repair patients and CPET-stratified conservatively managed individuals did not show significance (Figure $4 \mathrm{c}$ ) at 45 months (logrank test: $P=0.62$ ).

\section{Non-operated patients (CPET era)}

Of the 230 patients identified with elective infra-renal AAA following the introduction of CPET at UHCW NHS Trust, 61 (26.5\%) had not undergone surgery prior to closure of the study. Within this group, 19 subjects were pending open or endovascular intervention (that is, on the waiting list) and $42(18.3 \%)$ were managed conservatively. Conservative management was principally in

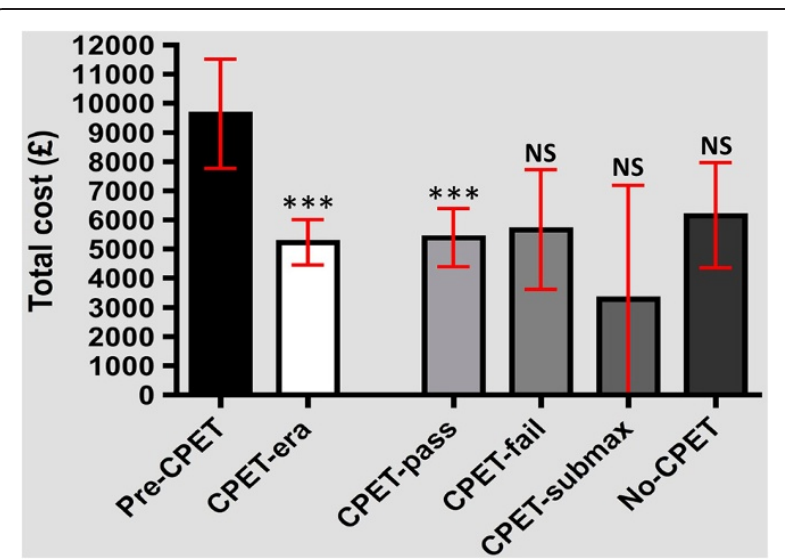

Figure 3 One-way ANOVA of total cost of inpatient stay for open AAA repairs in the pre-CPET and CPET eras. The four bars on the right represent the CPET-outcome breakdown for patients from November 2007 to the present. AAA: abdominal aortic aneurysm; ANOVA: analysis of variance; CPET: cardiopulmonary exercise testing; NS: not significant (in comparison to the median value for the pre-CPET cohort); ${ }^{* * *}: \mathrm{P}<0.001$ (in comparison to the pre-CPET cohort).

respect of failed or submaximal CPET, reflected by the significantly lower mean AT for this cohort (Figure 5a) despite the normal distribution of AT for the entire 2007 to 2011 cohort (Figure 5b). However, in some cases, alternate non-cardiorespiratory co-morbidities (e.g. ongoing malignancy), poor quality of life or loss of independence were quoted as indications for non-operative treatment. Two individuals chose to be managed conservatively despite an adequate AT for open intervention (Figure 1).

Conservatively managed individuals from the CPET era exhibited comparable all-cause mortality $(28.6 \%)$ to the unstratified pre-CPET control group (41.4\%; $P=0.15$ ). However, all-cause mortality for operated patients in the CPET era was significantly lower than those treated conservatively. These findings hold true for open and endovascular repair and were reflected only in CPET-pass patients on subgroup analysis (Table 5). A summary of allcause mortality for conservatively managed patients is shown in Table 6 . This reduction in all-cause mortality translates into a significant survival advantage for CPETstratified operated patients compared to conservatively managed individuals over the studied period (Figure 6; logrank test (curve comparison): $P<0.05$ ).

\section{Discussion}

Since the advent of CPET stratification for elective AAA patients at UHCW, a significant reduction in 30-day perioperative mortality rate (4\%; Table 4 ) for all open repairs $(n=100)$ has been noted. Individuals achieving $\mathrm{AT} \geq 11 \mathrm{ml} / \mathrm{kg} / \mathrm{min}$ upon CPET who subsequently underwent open AAA repair ( $n=74 / 100$ open repairs) exhibited $2.7 \%$ perioperative mortality, exceeding the 
Table 4 Fisher's exact test comparison of total 30-day mortality

\begin{tabular}{|c|c|c|c|c|}
\hline Cohort & & 30-day mortality (\%) & Odds ratio $(95 \% \mathrm{Cl})$ & $P$ value \\
\hline & Open surgery & & & \\
\hline Pre-CPET (Jan 03 to Oct 07) & Pre-CPET $(n=103)$ & 12.6 & & \\
\hline \multirow[t]{5}{*}{ CPET era (Nov 07 to Jul 11) } & CPET era (total) $(n=100)$ & 4.0 & $\mathbf{0 . 2 9}$ (0.09 to 0.92$)$ & $P<0.05$ \\
\hline & CPET-pass (74/100) & 2.7 & $\mathbf{0 . 1 9}$ (0.04 to 0.88 ) & $P<0.05$ \\
\hline & CPET-fail (8/100) & 12.5 & 0.989 (0.11 to 8.70$)$ & $P=1.00$ \\
\hline & CPET-submaximal (3/100) & 33.3 & 2.31 (0.22 to 23.90) & $P=0.43$ \\
\hline & No-CPET $(15 / 100)$ & 0 & 0.18 (0.01 to 3.20$)$ & $P=0.21$ \\
\hline
\end{tabular}

targets of the VSGBI QIF for 2013 [31] and this was a statistically significant reduction when compared to preCPET controls. By comparison, patients with AT $<11$ who proceeded nonetheless to open repair $(n=8 / 100)$ experienced equivalent 30 -day mortality $(12.5 \% ; P=1.00)$ to the unstratified pre-CPET cohort. Individuals undergoing open AAA repair despite being unable to generate AT during CPET $(n=3 / 100)$ witnessed $33.3 \%$ perioperative mortality $(P=0.43$ when compared to the pre-CPET era cohort). No perioperative deaths were reported following open AAA repair among the 15 individuals who were submitted for surgery without CPET; however this did not achieve statistical significance $(P=0.21)$ when compared to pre-CPET era controls.

A pre-operative AT $\geq 11 \mathrm{ml} / \mathrm{kg} / \mathrm{min}$ was also associated with reduced total LOS following open repair $(P<0.01)$ and EVAR $(P<0.05)$. Of interest, the 15 individuals submitted for open surgery without CPET also demonstrated a significant reduction in total LOS $(P<0.001)$ when compared to pre-CPET controls. Conversely, the 27 individuals who underwent EVAR without CPET risk stratification did not show such a reduction in $\operatorname{LOS}(P=0.14)$.

Following open surgery, the CPET-pass subgroup also benefited from reduced ITU LOS $(P<0.001)$ and median number of end organs supported $(P<0.001)$, which were not observed among individuals with $\mathrm{AT}<11$, no AT, or those who were not referred for CPET.

The reduction in length of inpatient stay demonstrated for both open and endovascular AAA repair may have considerable beneficial financial implications for trusts offering pre-operative CPET stratification.

EVAR patients exhibited a reduction in median length of stay from 6 to 4 days following the introduction of testing; hence there was a proportionate reduction in nonoperative costs attributable to fewer bed-nights on general surgical wards. Confounding factors in this analysis include a shift from pre-discharge (inpatient) computed tomography assessment of stent-graft position prior to 2007, to 30-day surveillance as an outpatient in more recent times. This may contribute to the reduction in inpatient stay witnessed within this cohort, although of note, the reduction in bed-nights required was only significant for individuals with AT $\geq 11$ on subgroup analysis. EVAR was associated with significantly shorter durations of inpatient stay, an almost abolished requirement for critical care services and lower mortality than open surgery, consistent with established work [30,36,37]. Thus, the witnessed increase in the proportion of EVAR cases should intuitively reduce overall elective AAA costs. However, the high cost of technologically advanced endovascular stents for such cases effectively abrogates this benefit when compared to open AAA repairs for individuals of adequate cardiorespiratory fitness [30,38].

A marked financial benefit was seen in open AAA repairs. Non-operative (fully absorbed) inpatient costs for the CPET-pass subgroup were approximately half of those for the pre-CPET era; there was an average saving of over $£ 4,000$ per patient. The most influential factors in this calculation included the significant reduction in ITU bed-nights utilized by AT $\geq 11$ patients in addition to a lower (median) number of end organs requiring support, thereby reducing the nightly critical care tariff. Thus, pre-operative CPET risk stratification appears to be a financially advantageous method for improving perioperative outcomes in elective aortic surgery. The non-operative cost savings in open AAA surgery alone may allow generation of revenue from such testing in the longer term. An efficient CPET service should be readily achievable within the modern vascular unit; this technology having been successfully transported, assembled and utilized in a field laboratory on the South Col of Mt. Everest [39], 8,000 m above sea level.

All-cause mortality for the CPET-era cohort (2007 to 2011) was shown to be greater among individuals managed conservatively (28.6\%; Tables 5 and 6) following CPET risk stratification, when compared to individuals concurrently submitted for open or endovascular surgery (6.5\%; $P<0.001)$. A significant survival advantage was confirmed for surgically managed patients compared with those treated conservatively (for the period studied) according to Kaplan-Meier analysis (Figure 6). Individuals within this subgroup were shown to have poorer cardiorespiratory fitness than those within the operative subgroups (Figure 5a, mean AT $9.2 \mathrm{ml} / \mathrm{kg} / \mathrm{min}$ c.f. $13.3 \mathrm{ml} / \mathrm{kg} / \mathrm{min}$; 


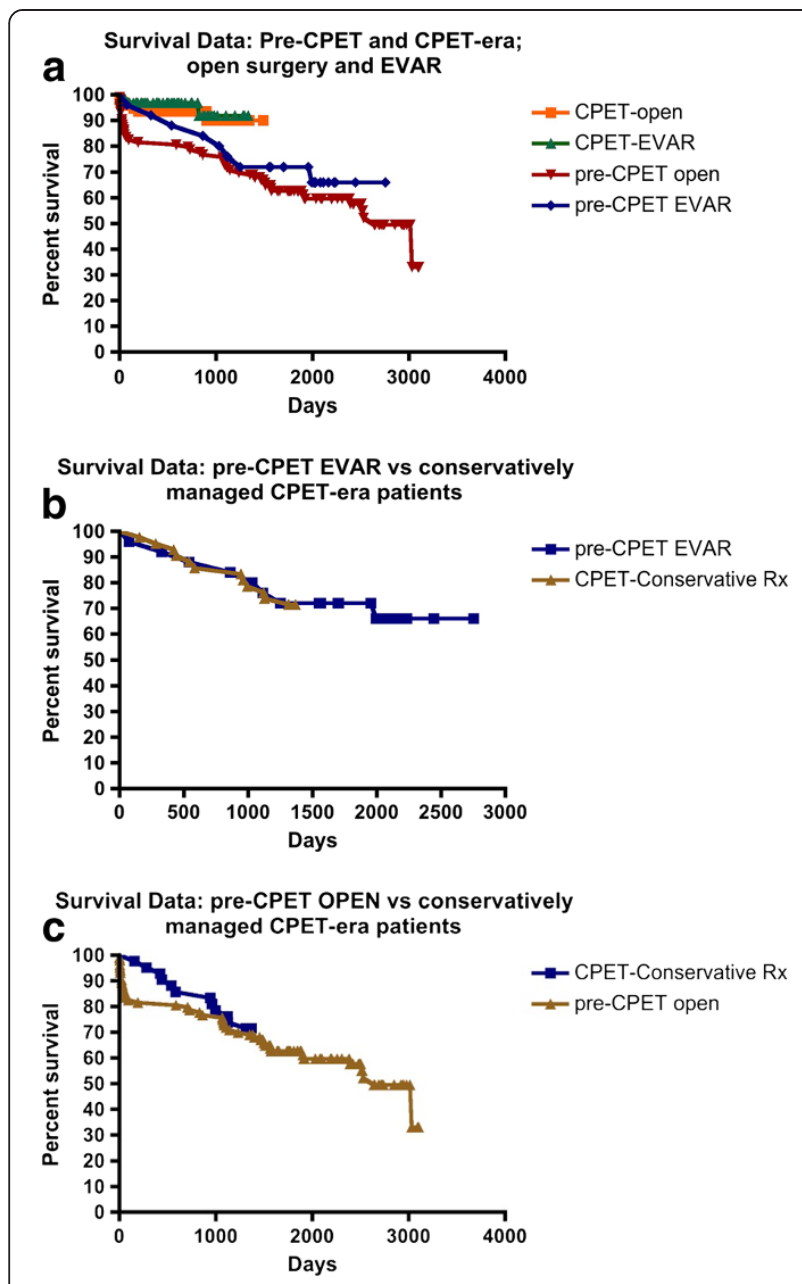

Figure 4 Kaplan-Meier survival analysis (all-cause mortality). (a) Comparison of open AAA repair and EVAR in the pre-CPET and CPET eras. (b) Comparison of pre-CPET EVAR subjects compared with CPET-era patients managed conservatively. Curve comparison by the logrank test demonstrated no significant difference $(P=0.96)$. Patients on the waiting list for aortic intervention (open or endovascular) at the close of the study have been removed from the analysis. (c) Comparison of pre-CPET open AAA repair compared with CPET-era patients managed conservatively. Curve comparison by logrank test demonstrated no significant difference $(P=0.62)$. Patients on the waiting list for aortic intervention (open or endovascular) at the closing date of the study have been removed from the analysis. AAA: abdominal aortic aneurysm; CPET: cardiopulmonary exercise testing; EVAR: endovascular aneurysm repair; Rx: management/treatment.

$P<0.001)$. Data for the conservatively treated subgroup (Table 6) suggest mortality predominantly resulted from significant underlying co-morbidities and supports a nonoperative approach, consistent with the findings of previous studies [40]. Known mortality from ruptured AAA for the conservatively managed subgroup was $2.4 \%$, within the limitations of this retrospective study. By implication, therefore, only a minority of deaths within this subgroup could have been prevented by intervention (open or en-

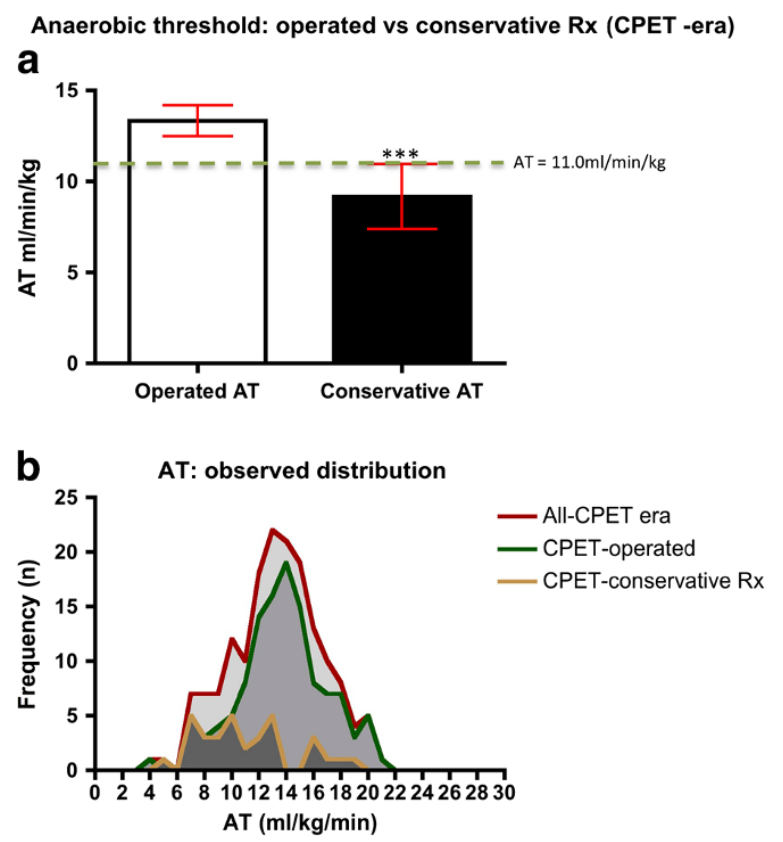

Figure 5 Anaerobic threshold. (a) Mann-Whitney $U$ comparison of mean $(95 \% \mathrm{Cl})$ for CPET stratified and subsequently operated patients (13.3 ml/kg/min, $95 \%$ Cl: 12.5 to 14.2 ) with those managed conservatively (9.2 ml/kg/min, 95\% Cl: 7.2 to 10.9:*** $P<0.001$ ). The dashed line delineates the evidence-based threshold of $11.0 \mathrm{ml} / \mathrm{kg} / \mathrm{min}$. (b) Anaerobic threshold distributions for all CPET patients ( $n=166$, normal distribution on D'Agostino and Pearson omnibus normality test), CPETera operated ( $n=118$, skewed distribution) and CPET conservatively managed subjects ( $n=33$, skewed distribution). Submaximal test results (no AT) have been removed from this analysis. AT: anaerobic threshold; CPET: cardiopulmonary exercise testing; Rx: management/treatment.

dovascular AAA repair). However, for an individual of poor cardiorespiratory status, such an intervention would have been associated with a high risk of perioperative complications and mortality [36,40]; that is, death or serious morbidity may have been hastened by surgery. Longer-term follow-up would be required, ideally within the confines of a prospective study, to ascertain the natural progression of CPET-stratified conservatively managed patients. Assessment of the frequency of AAA-related and other deaths, morbidity and survival may develop a clearer evidence base for the optimum management of unfit individuals.

A remaining equivocation relates to the degree of stringency with which pre-operative CPET stratification is applied as a gateway to open surgery. The findings of this study show that a dramatic reduction in 30-day mortality (12.6\% to $2.7 \%$ ) is achievable if an AT $\geq 11.0$ is considered an absolute requirement. By contrast CPETguided pre-operative decision-making (allowing for clinical discretion) resulted in 4\% 30-day mortality and permitted surgical management of a further 26 individuals. Ultimately, individual vascular units offering this service 
Table 5 Fisher's exact test comparison of all-cause mortality

\begin{tabular}{lllll}
\hline Cohort & Group & All-cause deaths & $P$ value & Odds ratio \\
\hline CPET era & Conservative Rx $(n=42)$ & $12(28.6 \%)$ & & 0.15 \\
Pre-CPET & All $(n=128)$ & 53 & 0.10 & N/A \\
& Open $(n=103)$ & 45 & 0.79 & N/A \\
& EVAR $(n=25)$ & 8 & $<0.001$ & $0.17(0.07$ to 0.43$)$ \\
CPET era & All operated $(n=169)$ & $11(6.5 \%)$ & $<0.01$ & $0.22(0.08$ to 0.58$)$ \\
& All open $(n=100)$ & $8(8 \%)$ & $<0.01$ & $0.22(0.08$ to 0.64$)$ \\
& CPET-pass OPEN $(n=74)$ & $6(8.1 \%)$ & $<0.001$ & $0.11(0.03$ to 0.43$)$ \\
& All EVAR $(n=69)$ & $3(4.3 \%)$ & $<0.01$ & $0.05(0.005$ to 0.85$)$ \\
\hline
\end{tabular}

CPET: cardiopulmonary exercise testing; EVAR: endovascular aneurysm repair; Rx: management/treatment.

will have to choose between optimizing perioperative mortality, or allowing patients of borderline cardiorespiratory fitness the chance of an elective AAA repair. This controversy, perhaps, warrants high-level debate as we move towards the 2013 target of 3.5\% 30-day mortality, as set by the VSGBI QIF [31].

Patients undergoing open or endovascular AAA repair in the pre-CPET era exhibited reduced mid-term survival when compared to the CPET-era operated cohorts. Indeed, at 45 months no significant survival advantage was conferred to the unstratified open surgery or EVAR patients, when compared to CPET-stratified conservatively managed individuals (Figure 4b,c). For pre-CPET EVAR patients, who demonstrated negligible perioperative mortality rates, a prevalence of significant underlying co-morbidities in subjects selected for this intervention may contribute to these data, consistent with the findings of Goodney et al. [41]. The less metabolically demanding EVAR is routinely offered to carefully selected patients who fail to achieve a satisfactory AT on exercise testing. Equivalent survival between these patients and conservatively managed individuals, again, suggests a requirement for further study; conservative management may be more appropriate for such

Table 6 A summary of all-cause mortality in nonoperated patients following CPET stratification

\begin{tabular}{llll}
\hline Cohort & Group & Deaths & Frequency \\
\hline $\begin{array}{l}\text { CPET era } \\
(n=230)\end{array}$ & Conservatively & Total & $12(28.6 \%)$ \\
& & $\begin{array}{l}\text { Communed }(n=42) \\
\text { (unknown cause) }\end{array}$ & $6(14.3 \%)$ \\
& $\begin{array}{l}\text { Ruptured AAA } \\
\text { Cardiorespiratory }\end{array}$ & $1(2.4 \%)$ \\
& disease & $1(2.4 \%)$ \\
& Malignancy & $2(4.8 \%)$ \\
& Sepsis & $2(4.8 \%)$
\end{tabular}

AAA: abdominal aortic aneurysm; CPET: cardiopulmonary exercise testing. patients [40], based upon specific morbidities, quality-of -life outcomes and mode of death.

For pre-CPET-era open AAA repairs, the finding of equivalent survival at 45 months (Figure $4 \mathrm{c}$ ) when compared to CPET-stratified individuals rejected for surgery, strongly reflects the perioperative death rate. This is suggested by the significantly reduced perioperative mortality rates for CPET-stratified open AAA patients and the concurrent mid-term survival advantage seen for this Cohort (Figure 4a). Moreover, the improved survival trend for CPET-stratified open AAA patients suggests that their superior cardiorespiratory fitness (Figure 5a) may preserve the survival advantage in the longer term and should be subject to further study.

\section{Limitations of the study}

Compliance with the trust-wide guideline of pre-operative CPET for all elective AAA patients was incomplete, with 42 (18.3\%) patients submitted for surgery (15 open repairs and 27 EVAR) without testing. Moreover, an element of cross-over was permitted following risk stratification;

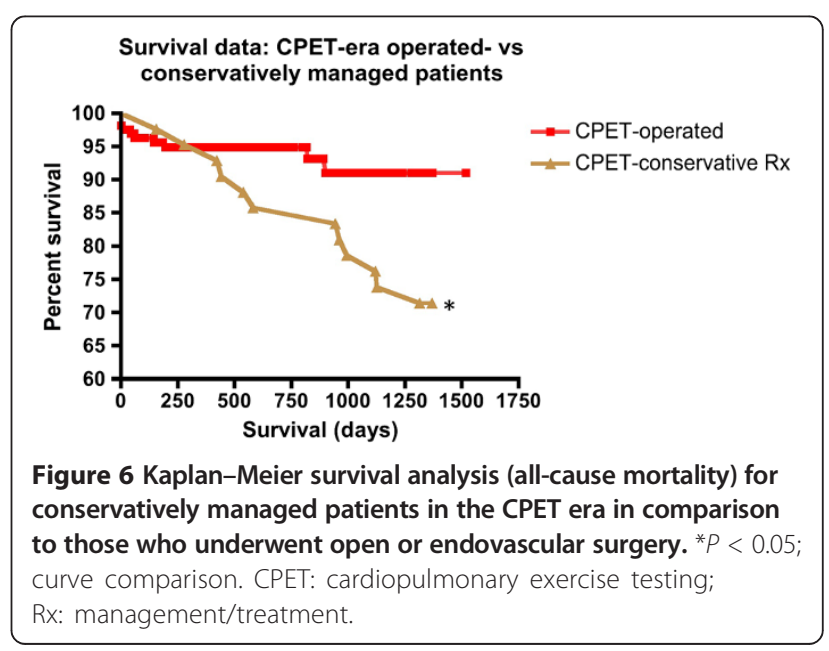


individuals within the CPET-fail $(\mathrm{AT}<11)$ or CPETsubmaximal (no AT) subgroups were not prevented from proceeding to open surgery upon request. A number of patients were disinclined to manage their AAA expectantly (when consideration was given to the accepted 90\% mortality following rupture [32]), instead they accepted the increased risk of perioperative mortality and proceeded to intervention. Morally, this was a difficult view to oppose. Such limitations of this retrospective study dictated a need for subgroup analysis of the open repair and EVAR patient groups within the CPET-era cohort (see Figure 1) to avoid confounding results.

Furthermore, despite exhaustive efforts to identify all patients with elective infra-renal AAA within both cohorts, no conservatively managed individuals could be detected in the pre-CPET era. Notwithstanding the trust's all-comers policy to aneurysm repair prior to 2007, the authors are reluctant to accept that there were no such patients. By implication, a degree of misclassification and data loss has to be assumed (leading to information bias). Such bias is compensated in part, by the subgroup analyses performed. However, potentially valuable comparisons between subjectively determined conservatively managed individuals of the pre-CPET era and those objectively identified by CPET, were not possible.

\section{Conclusion}

The introduction of pre-operative CPET risk stratification for elective AAA repair patients (as a quality improvement strategy) has shown improved perioperative outcomes.

In this retrospective study, an anaerobic threshold of $\geq 11.0 \mathrm{ml} / \mathrm{kg} / \mathrm{min}$ has been positively associated with reduced perioperative (30-day) mortality, total LOS, length of ITU stay and support requirements for open surgical patients. As a consequence, non-operative costs were significantly reduced for these individuals. For EVAR patients, AT $\geq 11.0$ was similarly associated with a reduced total LOS and thus, non-operative costs.

A significant mid-term survival advantage is also seen for CPET-stratified open repair and EVAR cohorts over controls, consistent with previous findings [26]. A consequence of CPET stratification (and clinical discretion) was the generation of a conservatively managed, unfit patient cohort. These individuals demonstrated greater all-cause mortality than surgically managed patients, principally of non-aneurysmal etiology, justifying the non-operative approach.

\section{Recommendations for further study}

A prospective randomized controlled trial (RCT) would be scientifically most appropriate to confirm the findings of this study, potentially implicating CPET as an appropriate risk assessment tool contributing to mortality pre- diction in AAA surgery, as per NICE recommendations for further research [42]. A recently published study suggests that the paucity of robust data should preclude routine adoption of CPET in risk-stratifying patients undergoing major vascular surgery [15]. However, to the authors' knowledge, this manuscript describes the largest current single-center UK series of patients risk-stratified in this manner for elective AAA repair, with numerous potentially advantageous outcomes. Thus, there is precedent for a RCT to clarify this ongoing and controversial issue.

\section{Abbreviations \\ AAA: Abdominal aortic aneurysm; ANOVA: Analysis of variance; AT: Anaerobic threshold; BMI: Body mass index; CPET: Cardiopulmonary exercise testing; CRRS: Clinical results reporting system; ECG: Electrocardiogram; \\ EVAR: Endovascular aneurysm repair; FEV $_{1}$ : Forced expiratory volume in 1 second; FVC: Forced vital capacity; LOS: Length of inpatient stay; ITU: Intensive therapy unit; MET: Metabolic equivalent; NHS: National Health Service; NICE: National Institute for Health and Care Excellence; NS: Not significant; QIF: Quality improvement framework; RCT: Randomized controlled trial; Rx: Management/treatment; UHCW: University Hospitals Coventry and Warwickshire; VSGBI: Vascular Society of Great Britain and Ireland.}

\section{Competing interests}

The authors declare that they have no competing interests.

\section{Authors' contributions}

SJG: data collection, interpretation, analysis, drafting and revision of manuscript, intellectual content of study. HY: concept and design, data collection. MS: data collection and analysis. JS: data collection, drafting of manuscript, critical revisions. CH: data collection. DW: data analysis, interpretation, critical revisions for intellectual content. CM: revision of manuscript, intellectual content. AM: revision of manuscript, intellectual content. DH: concept, intellectual content. CHEl: concept and design, data interpretation, drafting and revision of manuscript, intellectual content of study. All authors read and approved the final manuscript.

\section{Authors' information}

SJG: MBChB MD MRCS. SpR in Vascular/General Surgery, West Midlands Deanery. HY: MBChB FRCS. SpR in Vascular/General Surgery, West Midlands Deanery. MS: MBChB MRCS. Research Registrar in Vascular Surgery, UHCW NHS Trust. JS: MSC BSC. Clinical Service Manager, Department of Respiratory Physiology, University Hospitals Coventry and Warwickshire NHS Trust.

CEH: MBChB MRCS. SpR in Trauma and Orthopedics. West Midlands Deanery. DW: FRCA. Consultant in Critical Care and Anesthetics. Medical Lead Central England Critical Care Network and Chair of the National Forum of Medical Leads for Critical Care Networks.

CM: MBBS FRCS. Consultant Vascular Surgeon. UHCW NHS Trust.

AM: MD FRCS. Consultant Vascular and Endovascular Surgeon, UHCW NHS Trust. DH: MS MMedEd FRCS. Consultant Vascular Surgeon and Clinical Lead, UHCW NHS Trust.

CHEl: (Professor) PhD FRCS FRCP. Director of Research and Development, Associate Medical Director. Consultant Vascular, Endovascular and Renal Transplant Surgeon

Warwick Medical School and University Hospitals Coventry and Warwickshire NHS Trust.

\section{Collaborators}

Andrew Taylor (Finance Manager, Surgical Directorate, UHCW NHS Trust), Andrew Roberts (Information Systems Manager Department of Critical Care, UHCW NHS Trust), Julie Aughton (Specialist Respiratory Physiologist), Mr P Blacklay and Mr K Zayyan (Consultant Vascular Surgeons), Dr A Scase, Dr A Thacker, Dr E Borman, Dr B Dudkovsky, Dr S Sreevathsa and Dr A Kelly (Consultant Anesthetists). 


\section{Funding}

This research received no specific grant from any funding agency in the public, commercial or not-for-profit sectors.

\section{Author details}

${ }^{1}$ University Hospitals Coventry and Warwickshire NHS Trust, Clifford Bridge Road, Coventry CV2 2DX, UK. ${ }^{2}$ Warwick Medical School, University of Warwick, Coventry CV4 7AL, UK.

Received: 19 July 2012 Accepted: 18 April 2013

Published: 19 May 2013

\section{References}

1. Waxman K: Hemodynamic and metabolic changes during and following operation. Crit Care Clin 1987, 3(2):241-250.

2. Silverstein PR, Caldera DL, Cullen DJ, Davison JK, Darling RC, Emerson CW: Avoiding the hemodynamic consequences of aortic cross-clamping and unclamping. Anesthesiology 1979, 50(5):462-466

3. Attia RR, Murphy JD, Snider M, Lappas DG, Darling RC, Lowenstein E: Myocardial ischemia due to infrarenal aortic cross-clamping during aortic surgery in patients with severe coronary artery disease. Circulation 1976, 53(6):961-965.

4. Pearson S, Hassen T, Spark Jl, Cabot J, Cowled P, Fitridge R: Endovascular repair of abdominal aortic aneurysm reduces intraoperative cortisol and perioperative morbidity. J Vasc Surg 2005, 41(6):919-925.

5. Salatash K, Sternbergh WC, York JM, Money SR: Comparison of open transabdominal AAA repair with endovascular AAA repair in reduction of the postoperative stress response. Ann Vasc Surg 2001, 15:53-59.

6. Baxendale BR, Baker DM, Hutchinson A, Chuter TA, Wenham PW, Hopkinson BR: Haemodynamic and metabolic response to endovascular repair of infra-renal aortic aneurysms. Br J Anaesth 1996, 77(5):581-585.

7. Struthers R, Erasmus P, Holmes K, Warman P, Collingwood A, Sneyd JR: Assessing fitness for surgery: a comparison of questionnaire, incremental shuttle walk, and cardiopulmonary exercise testing in general surgical patients. Br J Anaesth 2008, 101(6):774-780

8. Shoemaker WC, Appel PL, Kram HB, Waxman K, Lee TS: Prospective trial of supranormal values of survivors as therapeutic goals in high-risk surgical patients. Chest 1988, 94(6):1176-1186.

9. Older P, Smith R: Experience with the preoperative invasive measurement of haemodynamic, respiratory and renal function in 100 elderly patients scheduled for major abdominal surgery. Anaesth Intensive Care 1988, 16(4):389-395.

10. Buck N, Devlin HB, Lunn JN: The Report of a Confidential Enquiry into Perioperative Deaths. London: The Nuffield Provincial Hospital Trust and the Kings Fund; 1987.

11. Eagle KA, Berger PB, Calkins H, Chaitman BR, Ewy GA, Fleischmann KE, Fleisher LA, Froehlich JB, Gusberg RJ, Leppo JA, Ryan T, Schlant RC, Winters WL Jr, Gibbons RJ, Antman EM, Alpert JS, Faxon DP, Fuster V, Gregoratos G, Jacobs AK, Hiratzka LF, Russell RO, Smith SC Jr: ACC/AHA guideline update for perioperative cardiovascular evaluation for noncardiac surgery executive summary: a report of the American College of Cardiology/ American Heart Association Task Force on Practice Guidelines (Committee to Update the 1996 Guidelines on Perioperative Cardiovascular Evaluation for Noncardiac Surgery). J Am Coll Cardiol 2002, 39(3):542-553.

12. Aronow WS, Ahn C: Incidence of heart failure in 2,737 older persons with and without diabetes mellitus. Chest 1999, 115(3):867-868.

13. Reilly DF, McNeely MJ, Doerner D, Greenberg DL, Staiger TO, Geist MJ, Vedovatti PA, Coffey JE, Mora MW, Johnson TR, Guray ED, Van Norman GA, Fihn SD: Self-reported exercise tolerance and the risk of serious perioperative complications. Arch Intern Med 1999, 159(18):2185-2192.

14. Fleisher LA, Beckman JA, Brown KA, Calkins H, Chaikof EL, Fleischmann KE, Freeman WK, Froehlich JB, Kasper EK, Kersten JR, Riegel B, Robb JF, Smith SC Jr, Jacobs AK, Adams CD, Anderson JL, Antman EM, Buller CE, Creager MA, Ettinger SM, Faxon DP, Fuster V, Halperin JL, Hiratzka LF, Hunt SA, Lytle BW, Nishimura R, Ornato JP, Page RL, Riegel B, et al: ACC/AHA 2007 Guidelines on Perioperative Cardiovascular Evaluation and Care for Noncardiac Surgery: Executive Summary: A Report of the American College of Cardiology/American Heart Association Task Force on Practice Guidelines (Writing Committee to Revise the 2002 Guidelines on Perioperative Cardiovascular Evaluation for Noncardiac Surgery)
Developed in Collaboration With the American Society of Echocardiography, American Society of Nuclear Cardiology, Heart Rhythm Society, Society of Cardiovascular Anesthesiologists, Society for Cardiovascular Angiography and Interventions, Society for Vascular Medicine and Biology, and Society for Vascular Surgery. J Am Coll Cardiol 2007, 50(17):1707-1732.

15. Young EL, Karthikesalingam A, Huddart S, Pearse RM, Hinchliffe RJ, Loftus IM, Thompson MM, Holt PJ: A systematic review of the role of cardiopulmonary exercise testing in vascular surgery. Eur J Vasc Endovasc Surg 2012, 44:64-71.

16. Bauer SM, Cayne NS, Veith FJ: New developments in the preoperative evaluation and perioperative management of coronary artery disease in patients undergoing vascular surgery. J Vasc Surg 2010, 51(1):242-251.

17. Thompson AR, Peters N, Lovegrove RE, Ledwidge S, Kitching A, Magee TR, Galland RB: Cardiopulmonary exercise testing provides a predictive tool for early and late outcomes in abdominal aortic aneurysm patients. Ann $R$ Coll Surg Engl 2011, 93(6):474-481.

18. Franciosa JA, Park M, Levine TB: Lack of correlation between exercise capacity and indices of resting left ventricular ejection performance in heart failure. Am J Cardiol 1981, 47:33-39.

19. Lipkin DP: The role of exercise testing in chronic heart failure. Br Heart J 1987, 58:559-566

20. Smith TB, Stonell C, Purkayastha S, Paraskevas P: Cardiopulmonary exercise testing as a risk assessment in non cardio-pulmonary surgery: a systematic review. Anaesthesia 2009, 64:883-893.

21. Henry K, Gilliland C, Sharkey R, Daly JG, Kelly MG, Mc Cune K, et al: The use of cardiopulmonary exercise testing in the pre-operative assessment of patients awaiting abdominal aortic aneurysm repair in a District General Hospital. Irish J Med Sci 2010, 179:S496.

22. Kothmann E, Danjoux G, Owen SJ, Parry A, Turley AJ, Batterham AM: Reliability of the anaerobic threshold in cardiopulmonary exercise testing of patients with abdominal aortic aneurysms. Anaesthesia 2009, 64(1):9-13.

23. Older P, Smith R, Courtney P, Hone R: Preoperative evaluation of cardiac failure and ischaemia in elderly patients by cardiopulmonary exercise testing. Chest 1993, 104:701-704.

24. Older P, Hall A, Hader R: Cardiopulmonary exercise testing as a screening test for perioperative management of major surgery in the elderly. Chest 1999, 116:355-362

25. Snowden CP, Prentis JM, Anderson HL, Roberts DR, Randles D, Renton M, Manas DM: Submaximal cardiopulmonary exercise testing predicts complications and hospital length of stay in patients undergoing major elective surgery. Ann Surg 2010, 251(3):535-541.

26. Carlisle J, Swart M: Mid-term survival after abdominal aortic aneurysm surgery predicted by cardiopulmonary exercise testing. Br J Surg 2007, 94:966-969.

27. Hartley RA, Pichel AC, Grant SW, Hickey GL, Lancaster PS, Wisely NA, McCollum CN, Atkinson D: Preoperative cardiopulmonary exercise testing and risk of early mortality following abdominal aortic aneurysm repair. Br J Surg 2012, 99(11):1539-1546.

28. Scott RAP, Ashton HA, Buxton MJ, Day NE, Day NE, Marteau TM, et al: The Multicentre Aneurysm Screening Study (MASS) into the effect of abdominal aortic aneurysm screening on mortality in men: a randomised controlled trial. Lancet 2002, 360:1531-1539.

29. The UK Small Aneurysm Trial Participants: Mortality results for randomised controlled trial of early elective surgery or ultrasonographic surveillance for small abdominal aortic aneurysms. Lancet 1998, 352:1649-1655.

30. EVAR trial participants: Comparison of endovascular aneurysm repair with open repair in patients with abdominal aortic aneurysm (EVAR trial 1), 30-day operative mortality results: randomised controlled trial. Lancet 2004, 364:844-848.

31. Vascular Society of Great Britain and Ireland: UK National AAA Quality Improvement Framework document, 2009 - Updated 2011. [http://www.vascularsociety.org.uk/vascular/wp-content/uploads/2012/ 11/NSGBI-AAA-QIF-2011-v4.pdf] (last accessed 24/05/2013).

32. Bengtsson $H$, Bergqvist D: Ruptured abdominal aortic aneurysm: a population-based study. J Vasc Surg 1993, 18:74-80.

33. Blankensteijn JD, Lindenburg FP, Van der Graaf Y, Eikelboom BC: Influence of study design on reported mortality and morbidity rates after abdominal aortic aneurysm repair. Br J Surg 1998, 85:1624-1630. 
34. National Research Ethics Service: Changes to the Remit of Research Ethics Committees; 2011. Available at www.nres.nhs.uk/EasySiteWeb/GatewayLink. aspx?alld=134047. Last accessed 05/05/2013.

35. Beaver WL, Wasserman K, Whipp BJ: A new method of detecting anaerobic threshold by gas exchange. J Appl Physiol 1986, 60:2020-2027.

36. Prentis JM, Trenell MI, Jones DJ, Lees T, Clarke M, Snowden CP: Submaximal exercise testing predicts perioperative hospitalization after aortic aneurysm repair. J Vasc Surg 2012, 56(6):1564-1570.

37. Shiels H, Desmond AN, Parimkayala R, Cahill J: The impact of abdominal aortic aneurysm surgery on intensive care unit resources in an Irish tertiary centre. Ir J Med Sci 2012.

38. Finlayson SR, Birkmeyer JD, Fillinger MF, Cronenwett JL: Should endovascular surgery lower the threshold for repair of abdominal aortic aneurysms? J Vasc Surg 1999, 29:973-985.

39. Levett DZH, Martin DS, Wilson MH, Mitchell K, Dhillon S, Rigat F, et al: Design and conduct of Caudwell Xtreme Everest: an observational cohort study of variation in human adaptation to progressive environmental hypoxia. BMC Med Res Methodol 2010, 10:98.

40. EVAR Trial Participants: Endovascular aneurysm repair and outcome in patients unfit for open repair of abdominal aortic aneurysm (EVAR trial 2): randomised controlled trial. Lancet 2005, 365:2187-2192.

41. Goodney PP, Travis L, Lucas FL, Fillinger MF, Goodman DC, Cronenwett IL, Stone DH: Survival after open versus endovascular thoracic aortic aneurysm repair in an observational study of the Medicare population. Circulation 2011, 358:464-474.

42. National Institute for Health and Care Excellence (NICE): technology appraisa guidance 167. Endovascular Stent Grafts for the Treatment of Abdominal Aortic Aneurysms. http://www.nice.org.uk/nicemedia/live/12129/43289/ 43289.pdf (last accessed 05/05/2013).

doi:10.1186/2047-0525-2-10

Cite this article as: Goodyear et al:: Risk stratification by pre-operative cardiopulmonary exercise testing improves outcomes following elective abdominal aortic aneurysm surgery: a cohort study. Perioperative Medicine 2013 2:10.

\section{Submit your next manuscript to BioMed Central and take full advantage of:}

- Convenient online submission

- Thorough peer review

- No space constraints or color figure charges

- Immediate publication on acceptance

- Inclusion in PubMed, CAS, Scopus and Google Scholar

- Research which is freely available for redistribution 